Development and Psychopathology, 15 (2003), 55-71

Copyright 2003 Cambridge University Press

\title{
Do aggressive/destructive toddlers lack concern for others? Behavioral and physiological indicators of empathic responding in 2-year-old children
}

\author{
KATHRYN L. GILL AND SUSAN D. CALKINS \\ University of North Carolina at Greensboro
}

\begin{abstract}
Ninety-nine 2-year-olds, out of a larger sample of 474 children, were classified as high $(n=49)$ or low $(n=50)$ in externalizing (aggressive/destructive) behaviors based on maternal reports assessed twice across a 2-month period. During a laboratory assessment, these toddlers participated in two empathy-eliciting tasks, from which affective, behavioral, and physiological measures were derived. Relations among measures of empathy were examined both within and across episodes and aggression groups. Analyses indicated that different indices of empathy were related to each other, both within and across empathy situations. In addition, aggressive children displayed more behaviors indicative of empathy than did nonaggressive children. Finally, a pattern of physiological responding to another's distress was evident across both groups of children, and some results indicated that greater physiological regulation was related to less empathy-related behavior. Results are discussed in terms of the developing nature of empathy and its changing association with both self-regulation and aggression.
\end{abstract}

Recently, there has been a great deal of interest in the role of emotional functioning in successful psychosocial adaptation (c.f. Hubbard \& Coie, 1994; Fox, Schmidt, Calkins, Rubin, \& Coplan, 1996; Thompson \& Calkins, 1996). Individual differences in how emotions are experienced, expressed, and reg-

This research was supported by a National Institute of Mental Health B/START award and a Research Council Grant and Summer Excellence Award from the University of North Carolina at Greensboro to Susan D. Calkins. Portions of this paper were presented at the Biennial Meeting of the International Society for Infant Studies, April 1998. The authors would like to thank Amy Clark, Susan Dedmon, Mary Johnson, Laura Lomax, and Cynthia Smith for their help in subject recruitment and in data collection and coding. The authors also thank the families who generously gave their time to participate in the study. Address correspondence and reprint requests to: Susan D. Calkins, Department of Psychology, P.O. Box 26164, University of North Carolina at Greensboro, Greensboro, NC 27412-6164; E-mail: sdcalkinn @ uncg.edu ulated have all been examined in relation to various indices of adjustment (Bates, Bayles, Bennet, Ridge, \& Brown, 1991; Campbell, 1990; Calkins, Gill, Smith, \& Johnson, 1999; Fox et al., 1996; Hubbard \& Coie, 1994). Although most of the research in this area has focused on directly experienced emotions, there is a growing interest in the area of vicariously induced emotions, specifically, the vicariously induced negative emotions surrounding the experience of empathy (e.g., Eisenberg et al., 1994, 1996; Eisenberg \& Fabes, 1995; Guthrie et al., 1997; ZahnWaxler, Cole, Welsh, \& Fox, 1995).

Although empathy may be viewed as an indicator of more general emotional functioning, it is clear that the ability to experience and express empathy when faced with the distress of others is integral to social development (Batson, 1991; Davis, 1994; Eisenberg \& Fabes, 1998; Eisenberg \& Miller, 
1987). The ability to express empathy has been associated with greater frequency of prosocial behaviors in social settings and higher ratings of success with peer groups (see Davis, 1994). Many theorist have argued that people who experience empathy will be more motivated to show helping behaviors or to stop aggressive behavior toward another person (e.g. Batson, 1991; Eisenberg \& Fabes, 1990; Hoffman, 1982). Moreover, there is growing evidence that these skills emerge relatively early.

There is evidence that a primitive form of empathy can be seen in the reactivity of newborns to the cries of another infant (Martin \& Clark, 1982; Sagi \& Hoffman, 1976), but more mature empathic responding is commonly accepted as appearing sometime during the 2nd year of life (Young, Fox, \& ZahnWaxier, 1999; Zahn-Waxler, Radke-Yarrow, Wagner, \& Chapman, 1992). Children begin to respond to the distress of others with otherfocused behaviors, such as concern, attention to the distress of another, cognitive exploration of the event, and prosocial interventions, during the 2 nd year of life. Although helping behaviors are rare in young children, particularly when a child is unfamiliar with the distressed other or there are other events competing for his or her attention (Howes \& Farver, 1987; Zahn-Waxler, Radke-Yarrow, et al., 1992; Zahn-Waxler, Robinson, \& Emde, 1992), prosocial behaviors appear and become more consistent during the 2 nd year of life. Other behavioral indicators of empathy occur with greater frequency in toddlerhood and may be observed in a child witnessing the distress of a relatively unknown adult. In the only series of studies of empathy development in toddlers, Zahn-Waxler and colleagues (Zahn-Waxler, Radke-Yarrow, et al., 1992; Zahn-Waxler, Robinson, \& Emde, 1992) reported measurable levels of concern, hypothesis testing (cognitive exploration of event), and arousal to the feigned distress of an examiner in the majority of participants as early as 20 months.

Although a great deal of attention has been given to the influence of empathy on helping and prosocial behaviors, less empirical consideration has been given to the relationship between empathy and aggressive and actingout behavior, especially in young children (see Davis, 1994). Some researchers have theorized that there should be a relation between aggressive behavior and a lack of empathy. These theorists point to the observation that the tendency to have low concern for the needs of other and the consequences of one's own actions is a common characteristic of disruptive behavior disorders (Zahn-Waxler et al., 1995). Reduced empathy, guilt, and concern toward others are common descriptors of externalizing behavior problems (American Psychiatric Association, 1994). However, in one review of the literature addressing the association between empathy and aggressive/ externalizing behavior, the relations were generally weak (Miller \& Eisenberg, 1988). The strongest associations were found when studies employed self-report measures rather than observation and when the participants were adolescents or adults. Many studies in which younger children were participants and observational measures of empathy were employed revealed weak or nonexistent relations between these two factors (Hastings, ZahnWaxier, Robinson, Usher, \& Bridges, 2000; MacQuiddy, Maise, \& Hamilton, 1987; Zahn-Waxler et al., 1995). It may be the case that low levels of empathic responding emerge over time in aggressive individuals. Alternatively, low numbers of aggressive subjects and measures that focus on more mature behaviors assumed to be associated with feelings of empathy, such as prosocial responding, could contribute to the low associations between high aggression and low empathy.

Aggression and empathy are theorized to be related through two possible mechanisms. First, aggressive behavior is commonly attributed, at least in older children and adults, to a characteristic underarousal that can be indexed physiologically (Raine, Venables, \& Mednick, 1997). Thus, a lack of empathy in aggressive individuals may be a consequence of a failure to be aroused to the distress of others. A recent study supports this hypothesis. Young and colleagues (1999) observed, in a study of temperamental contributors to empathy, that infants who displayed low motoric and affective arousal to novelty at 4 
months of age were less empathic at age 2 . However, there is little evidence in this study to suggest that these infants were likely to become aggressive. Van Hulle, Corley, ZahnWaxier, Kagan, and Hewit (2000) reported no relation between measures of physiological arousal as measured by resting heart rate (HR) and aggression from infancy to early childhood. In another study of 2-year-olds, Calkins and Dedmon (2000) observed that aggressive children displayed no physiological indicators of underarousal, as indexed by resting HR. They did find, however, that these children displayed poor behavioral and physiological regulation, as indexed by a lack of taskfocused behaviors and reduction of HR variability during challenging situations. Indeed, a second possible mechanism underlying the relation between empathy and aggression may be a failure of self-regulation.

A relative inability to regulate emotions and behaviors is characteristic of children with aggressive behavior problems (Campbell, 1990). From this perspective, low levels of empathy may be viewed as another type of regulatory deficit (Young et al., 1999), one that makes it difficult to attend and react appropriately to the emotions of others. For example, a child who struggles to regulate the empathic arousal felt in response to the distress of others may direct his attention to selfcomforting, rather than to the emotions and circumstances of the individual in distress. Therefore, two possible processes are hypothesized to underlie the association between aggression and empathy: low arousal and poor regulation. Both processes could function to produce low empathic responding in two different groups of individuals, one group that is unable to regulate empathic arousal and another that experiences little to no empathic arousal.

The hypotheses of underarousal and dysregulation lend themselves to a physiological investigation of empathy. Two general approaches have been adopted when investigating physiological responses in young children. The first approach involves finding stable physiological variables that are associated with stable personality traits and may be measured using a baseline or resting condition (c.f. Fox, 1989). The second approach involves examining specific changes in the physiological system in response to a specific emotional event (e.g., Calkins, 1997; ZahnWaxier et al., 1995). One area of physiological research investigating both resting and response measures of physiology is the study of cardiac activity. The HR and its inverse, heart period (HP), measure the activation of the sympathetic nervous system. The HR variability, or respiratory sinus arrhythmia (RSA), measures the variability in HR associated with breathing and may index an individual's competency to physiologically and behaviorally react to external stimuli (Porges, 1991; Porges, Doussard-Roosevelt, \& Maiti, 1994).

The second approach to the study of autonomic responses, involving measurement of physiological changes in response to emotionally arousing events, may be especially relevant to the study of empathy responses. Such responses involve competing demands of focusing on the self versus another and the management of negative affect experienced by the self in response to another's distress. These kinds of responses may be indexed by changes in cardiac activity in terms of both HR and HR variability. The direction and magnitude of change in HR has been linked in past research to different levels of social information processing (Cacioppo \& Sandman, 1978). Typically, deceleration of HR reflects attention directed outward (processing novel stimulation, for example), whereas acceleration reflects attention directed inward (during problem-solving conditions; Ruff \& Rothbart, 1996). In the area of empathy development, Eisenberg, Fabes, and their colleagues have examined specific changes in HR when subjects are presented with emotionally eliciting stimuli. Their work has suggested that deceleration of HR may be associated with the attention to others that characterizes empathy (Eisenberg, Fabes, Schaller, Carlo \& Miller, 1991). HR deceleration during exposure to the distress of others was also associated with an increase in desire to help (Eisenberg et al., 1989) and comforting (Fabes, Eisenberg, Karbon, Troyer, \& Switzer, 1994). On the other hand, HR acceleration has been associated with fear, self-focused regulation, and acting 
out (Cacioppo \& Sandman, 1978). This HR acceleration would indicate self-focused, personal distress, rather than empathy, in situations vicariously inducing negative emotionality. Changes in RSA during an empathyeliciting task may also indicate an attempt to regulate arousal (Porges, 1991; 1996). It has been suggested that RSA will decrease when a child's attention becomes more focused on external events (Calkins, 1997; Calkins \& Dedmon, 2000). Empathy is characterized by a child's attention to the distressing event and the victim. One would therefore expect to see empathy behaviors associated with a decrease in RSA during an empathy-eliciting task.

The present study was designed to investigate behavioral and physiological indices of empathy in a sample of 2 -year-old children with early-identified aggressive/destructive behavior problems. The assessment of children at this age was deemed appropriate to the study of both early empathy and aggressions for several reasons. This period has been identified as a critical one for the emergence of a number of important skills and milestones related to the development of an autonomous self and the necessary supporting self-regulatory skills (Belsky, Woodworth, \& Crnic, 1996; Edwards, 1995; Kopp, 1982; Sroufe, 1996). For example, by the time a child has reached the end of the toddler period, there is the expectation that he or she is capable of emotional and behavioral self-regulation skills that support an emergent independent identity and self-sufficient behavior (Cicchetti, Ganiban \& Barnett, 1991; Kopp, 1982). Moreover, autonomous and self-regulated behavior is viewed by some researchers as one index of early social competence (Campbell, 1997; Kopp, 1982). Thus, the toddler period is a key transition period for the development of independent functioning and a child's sense of agency, both of which underlie adaptive behavior during later childhood (Belsky et al., 1996), and the lack of which may be related to early problems with control of aggression (Campbell, 1990). The study of empathy behaviors and physiological responses in a sample of 2-year-olds with aggression/destructive tendencies may provide an empirical basis for the hypothesized links among empathy, regulation, and aggression. Moreover, the important question of whether relations between empathy and aggression that have been observed among older children extend downward developmentally lacks a clear answer. Other kinds of differences between aggressive and nonaggressive older children (notably, gender and physiological differences) are not observed in toddlerhood (Calkins \& Dedmon, 2000; Keenan \& Shaw, 1997; Van Hulle et al., 2000), leaving open the possibility that the relation between empathy and aggression may also differ in young children.

The specific aim of the present study was to examine situational empathy behaviors in 2 -year-olds identified as either high or low in aggressive/destructive behaviors. Previous studies suggest that older children who are aggressive toward others may by less empathic, are concerned less about distress in others, and may have difficulty regulating their emotions, including vicarious emotional experiences (Eisenberg \& Fabes, 1995; Eisenberg et al., 1996; Fabes et al., 1994). One important question is whether this association between uncontrolled/externalizing behaviors and empathy will be seen in younger children. Toward this end, children identified as aggressive/destructive by parents were observed in two empathy-eliciting situations. Following from Young and colleagues (Young et al., 1999), multiple dimensions of empathy behavior were measured and within and across task correlations were examined in an effort to characterize the kinds of responses that 2 year-olds display in such situations. A second issue that was addressed was whether there would be a physiological change in response to an empathy-eliciting situation that would reflect arousal and/or regulation. Based on previous research (Zahn-Waxler et al., 1995), it was hypothesized that 2-year-olds would display decreases in RSA and HP during the empathy-eliciting task, indicative of arousal and attempts to regulate it. Finally, relations between empathic behaviors and HP and RSA changes were examined. It was hypothesized that behaviors indicative of empathy, such as 
concern and curiosity about the causes of distress, would be associated with the decreases in RSA that are indicative of regulation.

Method

\section{Participants}

Four hundred seventy-four 2-year-old children ( $x=30$ months; 248 boys, 226 girls) from a southeastern city were recruited to take part in a questionnaire screening for behavior problems. Sixty-five percent of the families were European American, 30\% were African American, and the remaining 5\% were Asian, Hispanic or mixed race. The families were classified into socioeconomic status (SES) groups on the basis of employment information provided by the parents on the screening questionnaire. Sixty-one percent of the families were classified as middle class, $25 \%$ as lower class, and $14 \%$ as upper class. The racial and SES characteristics are representative of the county where recruitment took place. Parents were contacted through local child care centers, pediatricians' offices, and county health and human services facilities. Parents completed a behavior problem questionnaire, or were assisted in completing the form if they had reading difficulties, and a subset of the 474 children was selected for participation in the laboratory portion of the study. Procedures for selection of the target sample are described next.

Target sample selection. Of the larger screened sample a total of 121 children were initially selected for follow-up assessment on the basis of parents' responses to items on the Child Behavior Checklist (CBCL, 2-3 version, Achenbach, Edelbrock, \& Howell, 1987). To identify a group of children at high risk for problems with aggressive or destructive behaviors, the externalizing scale score (total score for aggressive and destructive subscales) for all 474 children was computed. Next, following from Achenbach (1992), a $t$ score cutoff of 60 was established; this represented the borderline clinical range. In Achenbach's (1992) study, children scoring in this range were 10 times more likely to have been referred for clinical services than children below this point. This cutoff represented the 80th percentile in the screened sample (the mean score for the entire screened sample was 52). As a contrast low-risk group, children whose $t$ score on the externalizing scale was 50 or below were selected. This represented the 50th percentile in the screened sample. Because not every child who was screened and met these criteria could be included in the study (e.g., the child was 3 years old by the time the questionnaire was scored, the family refused to participate or repeatedly missed appointments, or the family could not be contacted for an appointment) and because attempts were made to match the two risk groups in terms of race, SES, sex, and age, the initial selected sample consisted of 121 children (70 high risk, 51 low risk).

The high risk group was originally intended to have an $n$ of approximately 50 children. However, given the possibility that the screening process identified children with only transient behavior problems, a second assessment of externalizing problems was conducted when the parent and child came to the laboratory for the physiological and behavioral assessment, approximately 2 months later. Analysis of the two scores over the 2month period indicated that they were highly correlated $(r=.78, p<.0001)$. However, there was a significant decrease in the level of problem behavior among the high risk group, but not among the low risk group, $F(1)=22.01$, $p<.001$ (for the interaction term of Group $\mathrm{x}$ Time). For this reason, the selected sample of children was adjusted by using the mean of the two CBCL scores. Thus, the final sample consisted only of children whose mean score across the 2 -month period was 60 or above ( $n=49,24$ males) or 50 or below $(n=50,25$ males).

The two risk groups were matched on age ( $\boldsymbol{M} 32$ months for both groups, $S D=3.2$ months), SES ( $M=37$ for high risk and $M=$ 38 for low risk), race (34 Caucasians in the high risk group, 32 Caucasians in the low risk group), and maternal marital status (34 married in the high risk group, 39 married in the 
low risk group). There were differences between the groups on both measures (recruitment and assessment) of externalizing problem behavior ( $p<.001$ for both comparisons, $M=64.47$ and 62.57 , respectively, for the high risk group and $M=45.74$ and 44.32 , respectively, for the low risk group). However, there was no longer an interaction of time of assessment and group in terms of externalizing. In addition, there were no other differences between the two groups in terms of other sample characteristics (age, SES, race, marital status, and gender). Finally, there were no differences on any relevant measure (race, sex, age, SES, marital status, or problem score between children who met the behavior problem criteria for the study but did not participate and those who met the criteria and did participate. Nor were there any differences on these measures between the children who were dropped from the final sample because they did not have the appropriate mean CBCL score and those who were retained in the sample.

\section{Procedures}

The measures addressed in this study were part of a 2-hr laboratory assessment. During this visit, children and their mothers were asked to participate in tasks designed to measure affective, behavioral, and physiological emotionality and regulation; mother-child interaction behavior; and empathy behavior. The entire visit was filmed through a one-way mirror, for coding at a later time.

Upon arrival at the lab there was a brief warm-up period, after which the child was asked to sit at a table where three pediatric electrodes were placed on his or her chest and abdomen in an inverted triangle pattern. The electrodes were connected to a preamplifier, the output of which was transmitted to a vagal tone monitor (VTM-I, Delta Biometrics, Inc., Bethesda, MD) for R-wave detection. The vagal tone monitor detects $\mathrm{R}$-wave signals and displays ongoing HR and vagal tone (RSA) every $30 \mathrm{~s}$. A data file containing the interbeat intervals (IBIs) for the entire period of collection was transferred to a laptop computer for later artifact editing (resulting from child movement) and analysis. The experimenter used a marker button on the monitor to indicate the beginning and end of each HR collection episode. HR was collected during the first half of the assessment, after which the electrodes were removed and the child was free to move about the room.

While connected to the HR collection equipment, the child was observed during a six-episode sequence, only two of which (resting and Empathy Task 1) are relevant to the issues addressed in this article. The resting episode consisted of a 5-min segment of the videotape Spot, a short story about a puppy that explores its neighborhood. Although this episode was not a true resting measure of cardiac activity, given that the child's attention was engaged in an external stimuli, it was sufficient to keep the child sitting quietly and showing little affect. Given the ages of the subjects in this study, such a stimulus was necessary in order to keep the child seated at the table and to limit movement artifact in the HR data.

The following empathy-eliciting tasks were interspersed with positive interactions between the child and mother in order to eliminate any carryover effect.

Empathy Task 1: Crying tape. This task was an adaptation of a task by Martin and Clark (1982). The child was given a few simple picture books to look at while sitting at the table and connected to the HR recording equipment. The experimenter and mother appeared to be busy filling out forms. The mother continued to sit at the chair next to the child, and the experimenter sat a few feet away. An audiotape of another toddler crying was played outside the door to the playroom for a period of $2 \mathrm{~min}$. The experimenter continued to appear occupied unless the child questioned her about the crying, at which point she responded that the crying was coming from another child visiting the lab who was upset because his or her mother had left the room. The mother was instructed that if the child initiated an interaction with her, she should respond appropriately but should return to the questionnaires as soon as she could separate herself from the interaction. If the child asked 
about the crying or showed distress, the mother was told to respond with the same explanation that the experimenter used. The HR was measured throughout the task.

Empathy Task 2: Experimenter distress. This task was adapted from the work of ZahnWaxier and her colleagues (Zahn-Waxler, Radke-Yarrow et al., 1992). During this task, which occurred after the HR electrodes had been removed, the experimenter gave the child a small bucket of Duplo blocks to play with and then walked over to a closet on the opposite side of the room, where she pretended to injure her finger in the door. The experimenter knelt or sat on the floor and feigned distress, without making any eye contact with the child, for $45 \mathrm{~s}$ (e.g., "Ouch, I hurt my finger. It really hurts"). She then acted out a recovery phase for $15 \mathrm{~s}$ (e.g., "It feels better now"). The times for this task are slightly different from those of Zahn-Waxler and colleagues, who used two 30-s epochs. The injury episode was extended to $45 \mathrm{~s}$ to allow more time for the children to act. If the child attempted to interact with the experimenter, the experimenter ignored the child (acting more interested in her own distress) until the end of the episode. Several studies have successfully produced measurable empathy responding in young children, including children up to a year younger that the current sample, using these same procedures (Young et al., 1999; Zahn-Waxler, Radke-Yarrow et al., 1992). There were two female experimenters who recited identical scripts at a similar pace. Throughout the course of the study, the two experimenters repeatedly observed each other's performance to ensure uniform procedures. Additionally, the participants were randomly assigned to experimenters, and each experimenter saw similar numbers of children from each group. The mother remained on the sofa during this task and was instructed to ignore what was going on unless the child initiated contact with her. If the child went to the mother, she was instructed to limit her responses to the child to brief acknowledgments and not to suggest any action to the child. These maternal instructions were violated in less than $5 \%$ of the episodes.

\section{Measures}

Empathy coding. Videotapes of the empathy tasks were coded using a system adapted from the work of Zahn-Waxler, Robinson, and Emde (1992; Young et al., 1999) and included six measures from both tasks. This coding system does not attempt to separate empathy (a vicarious emotion similar to that of the victim) from sympathy (concern for the victim). Behaviors scored included:

1. Latency to respond: total time, in seconds, that it took a child from the beginning of the task to comment on, gesture toward, or approach the victim.

2. Self comforting: total duration of time spent at repetitive, physical, soothing behaviors such as thumb sucking, hair twirling, or stroking or pulling on some part of clothing or body.

3. Arousal: total duration of time spent in a state of high muscular arousal or freezing. Child is still with no gross motor movement and displays tension of rigidity.

4. Hypothesis testing: attempts to understand the victim's distress rated on a 5-point scale ( 1 = none; 2 = simple nonverbal gesture, e.g., one look from face to victims finger, or verbal probe, e.g., "you hurt finger?"; $3=$ combine a verbal and nonverbal inquiry; $4=2$ to 3 distinct attempts to understand; 5 = four or more inquires or lengthy explorations into cause of distress).

5. Concerned affect: expression of concern for victim through facial, gestural, or verbal displays of sadness rated on a 5-point scale ( 1 = none; 2 = slight or some concern, focused attention on victim with only fleeting brow furrowing, or a "drop" in facial affect; 3 = moderate concern expressed in face or voice, longer bouts of brow furrowing; 4 = moderate concern, prolonged brow furrowing or vocal contours express dismay for several seconds; 5 = great concern, sadness expressed, sympathy face, deep brow furrowing).

6. Global rating of empathy: combined information from previous codes to include con- 
cerned affect, prosocial behaviors, and involvement with the victim, rated on a 7 point scale $(1=$ no concern or interest $; 3=$ some concern; no prosocial behavior, but attentive; 5 = clear moderate concern; some prosocial action; 7 = clear consistent concern and prosocial behavior).

Two individuals who were blind to the risk status of the subjects became reliable on the described empathy coding. Ten percent of the original 121 subject tapes were coded jointly for training purposes, and an additional 10\% were coded independently in order to assess reliability. Data from both empathy tasks were included in one reliability score for each measure. The reliabilities for the duration codes range from $r=.85$ for arousal to $r=$ .94 for latency to respond to victim. Cohen's kappa for ordinal scales ranged from .70 for concerned affect to .86 for hypothesis testing.

Physiological changes during emotion eliciting tasks. To generate measures of cardiac activity from which to derive measures of resting heart period and RSA and heart period and RSA in response to the distress of another, the IBI files were edited and analyzed using MXEDIT software (Delta Biometrics, Bethesda, MD). Editing the files consisted of scanning the data for outlier points relative to adjacent data and replacing those points by dividing them or summing them so that they would be consistent with the surrounding data. Data files that required editing of more than $2 \%$ of the data ( 12 data points in a 5 -min period, for example) were not included in the analyses. Analysis of the IBI data consisted of applying the Porges (1985) method of calculating RSA. This method applies an algorithm to the sequential HP data. The algorithm uses a moving 21-point polynomial to detrend periodicities in HP slower than RSA. Then, a bandpass filter extracts the variance of $\mathrm{HP}$ within the frequency band of spontaneous respiration in young children, $0.24-1.04 \mathrm{~Hz}$. The estimate of RSA is derived by calculating the natural $\log$ (In) of this variance and is reported in units of $\operatorname{In}(\mathrm{ms}) 2$. The HP and RSA were calculated every $30 \mathrm{~s}$ for the 5 -min resting period and the 2-min empathy episode. The mean RSA and HP of the 30-s epochs within each episode were used in subsequent analyses of variance. In addition, RSA and HP change scores for the empathy episode were calculated by subtracting the RSA or HP during the empathy-eliciting episode from the corresponding measure from the resting episode; greater change scores for RSA and HP indicated greater decreases in the two measures in response to the distress of another. The data files of 16 children were not included in analyses involving physiological data. A few children would not allow the experimenter to apply the HR electrodes $(n=3)$. In addition, the HR data collection equipment failed on a few occasions $(n=4)$. However, the most common explanation for missing data was that the child pulled on the HR leads, which resulted in movement artifact affecting greater than $2 \%$ of the data in the HR file $(n=9)$. There was no relation between risk group membership and data loss.

\section{Results}

Prior to conducting the main analyses of the study, all behavioral measures were compared in terms of race and SES. No relations emerged between race or SES and any of the empathy measures. Next, the three issues outlined in the introduction were addressed. All correlations were initially tested separately by aggression group. However, in no case did the magnitude of any correlation differ by group, as tested using Fisher's $r$ to $\mathrm{z}$ transformation. A similar series of analyses were conducted by gender, and the magnitude of correlations did not differ by gender. Thus, all correlational analyses are reported for the entire sample. In addition, age was initially controlled for in all correlations. There was no change in the magnitude of correlations after controlling for age, so it was removed as a controlling factor in further analyses.

\section{Within-episode and cross-episode relations among empathy measures}

To characterize the empathy responses of 2year-olds and to examine whether there would be relations among measures within episodes, 
a correlation matrix was computed. These within-task correlations are presented in Table 1. As the table indicates, for the first empathy task, in which the child is exposed to, but cannot see, a crying toddler, there are significant correlations among many of the measures. Latency to respond, hypothesis testing, concern, and global empathy are all significantly correlated with each other. Arousal is also positively correlated with concern and global empathy but not with latency to respond or hypothesis testing. Self-comfort is only correlated with arousal. Thus, children who appear to be physically aroused, as indicated by behavioral freezing (Young et al., 1999), are also displaying more concern, self-comfort, and higher global empathy, but are not responding with the more responsive behaviors of hypothesis testing or short latencies to respond. Children who are responding quickly to the cries of another child and are doing more hypothesis testing are also demonstrating affective concern and global empathy. During the examiner distress tasks, latency to respond, hypothesis testing, concern, and global empathy were significantly correlated with each other but were unrelated to arousal or self-comfort. The relations among these four variables are similar to those seen in the crying tape episode, but arousal is not associated with other empathy behaviors seen during examiner distress.

Cross-episode relations were also examined for the six measures of empathy. These relations are also presented in Table 1 . As the table indicates, modest to strong correlations existed for most measures. Thus, children who responded empathically in Empathy Task 1 , as reflected in concern, hypothesis testing, latency to respond, and global empathy, were likely to respond empathically in Empathy Task 2. Self-comforting and arousal were not stable across episodes.

\section{Relations between empathy responding and aggressive/destructive behavior}

To examine whether there were significant differences in empathy behaviors (a) across empathy episodes, and (b) between aggressive/destructive and nonaggressive/destructive children a multivariate analysis of variance (MANOVA) was conducted using episode (two) and behavior (six) as within-subjects factors and aggression group as a betweensubjects factor. The overall MANOVA revealed a significant Episode $\mathrm{x}$ Behavior interaction effect, $F(5,465)=11.10, p<.001$, and a significant Group x Behavior interaction effect, $F(5,465)=5.22, p<.001$. The means for the six behaviors from both tasks for aggressive/destructive and nonaggressive/destructive children and the overall sample are presented in Table 2. This analysis indicates that across both episodes, there were differences across the entire sample in level of responding for some of the six behaviors and differences between the aggressive/destructive and nonaggressive-destructive children on some types of behaviors.

Follow-up MANOVAs were conducted on the six individual behaviors for the two tasks. These analyses indicated first that the Episode $\mathrm{x}$ Behavior effect was a function of the fact that the experimenter distress episode elicited greater arousal, $F(1,95)=5.40, p<$ .05 , more hypothesis testing, $F(1,95)=7.94$, $p<.01$, greater global empathy, $F(5,95)=$ $24.15, p<.001$, and shorter latencies to respond $F(5,95)=20.51, p<.001$, than did the crying tape. The Group $x$ Behavior analyses indicated that across both episodes, aggressive/ destructive children displayed shorter latencies to attend to the distress, $F(1,95)=3.95$, $p<.05$, more hypothesis testing $F(1,95)=$ $3.33, p<.10$, greater concern $F(1,95)=4.33$, $p<.05$, and greater global empathy, $F(1,95)=$ $9.47, p<.01$, than did nonaggressive children.

\section{Physiological responses to Empathy Task 1} (crying tape)

To examine whether there were significant differences in the physiological responses of RSA and HP (a) across episodes (two, resting and empathy), and (b) between aggressive/ destructive and nonaggressive/destructive children, two separate MANOVAs were conducted using episode as a within-subjects factor and aggression group and gender as between subjects factors. Gender was included as a between-subjects factor based on previ- 
Table 1. Correlations within and across episodes for measures of empathic responding $(\mathrm{n}=99)$

\begin{tabular}{|c|c|c|c|c|c|c|c|c|c|c|c|c|}
\hline & 1 & 2 & 3 & 4 & 5 & 6 & 7 & 8 & 9 & 10 & 11 & 12 \\
\hline \multicolumn{13}{|l|}{ Crying tape } \\
\hline 1. Latency & - & $-.82 * *$ & .03 & .10 & $-.27 * *$ & $-.57 * *$ & $.39 * * *$ &,$- 44 * * *$ & .08 & .06 & $-.32 * *$ & $-.32 * *$ \\
\hline 2. Hypothesis testing & & - & .12 & .04 & $.36 * * *$ & $.65^{* * *}$ & $-34 * * *$ & $.41 * * *$ & .05 & -.05 & $.30^{* *}$ & $.26^{* *}$ \\
\hline 3. Arousal & & & - & $.22 *$ & $.43^{* * * *}$ & $.28^{* *}$ & .02 & .02 & .05 & -.07 & .00 & .01 \\
\hline 4. Self-comfort & & & & - & .06 & .02 & .15 & -.16 & -.12 & .09 & $-.23 *$ & -.16 \\
\hline 5. Concern & & & & & - & $.87^{* * *}$ & .07 &, 09 & -.10 & $-.17 \dagger$ & $.28 * *$ & .09 \\
\hline $\begin{array}{l}\text { 6. Global empathy } \\
\text { Experimenter distress }\end{array}$ & & & & & & - & $-.18 \div$ & $.23 *$ & .09 & -.15 & $.34 * *$ & $.19 \dagger$ \\
\hline \multicolumn{13}{|l|}{ Experimenter distress } \\
\hline $\begin{array}{l}\text { 7. Latency } \\
\text { 8. Hypothesis testing }\end{array}$ & & & & & & & - & $-.82 * *$ & .05 & .04 & $-.48^{* * *}$ & $-.60 * * *$ \\
\hline 8. Hypothesis testing & & & & & & & & - & .04 & .00 & $.52 * * *$ & $.61 * * *$ \\
\hline 9. Arousal & & & & & & & & & - & .01 & .04 & .03 \\
\hline 10. Self-comfort & & & & & & & & & & - & .05 & .07 \\
\hline $\begin{array}{l}\text { 11. Concern } \\
\text { 12. Global empathy }\end{array}$ & & & & & & & & & & & - & $.79 * * *$ \\
\hline 12. Global empathy & & & & & & & & & & & & - \\
\hline
\end{tabular}

$t p<.10 .{ }^{*} p<.05 .{ }^{* *} p<.01 .{ }^{* * *} p<.001$. 
Table 2. Means and standard deviations of empathy measures for two tasks by aggression group and for the entire sample $(\mathrm{n}=99)$

\begin{tabular}{|c|c|c|c|c|c|c|}
\hline & \multicolumn{3}{|c|}{ Crying Tape } & \multicolumn{3}{|c|}{ Experimenter Distress } \\
\hline & $\begin{array}{c}\text { Low } \\
\text { Aggression }\end{array}$ & $\begin{array}{c}\text { High } \\
\text { Aggression }\end{array}$ & $\begin{array}{c}\text { Total } \\
\text { Sample }\end{array}$ & $\begin{array}{c}\text { Low } \\
\text { Aggression }\end{array}$ & $\begin{array}{c}\text { High } \\
\text { Aggression }\end{array}$ & $\begin{array}{l}\text { Total } \\
\text { Sample }\end{array}$ \\
\hline \multicolumn{7}{|c|}{ Latency (s) } \\
\hline M & 80.4 & 62.4 & 73.2 & 41.4 & 33.6 & 37.2 \\
\hline$S D$ & 52.8 & 51.6 & 52.8 & 23.4 & 20.4 & 23.4 \\
\hline \multicolumn{7}{|c|}{ Hypothesis testing } \\
\hline M & 2.58 & 2.79 & 2.67 & 2.80 & 3.30 & 3.04 \\
\hline$S D$ & 1.19 & 1.06 & 1.01 & 1.20 & 1.25 & 1.25 \\
\hline \multicolumn{7}{|c|}{ Arousal (prop.) } \\
\hline M & .03 & .01 & .02 & .06 & .05 & .06 \\
\hline$S D$ & .07 & .01 & .05 & .16 & .08 & .12 \\
\hline \multicolumn{7}{|c|}{ Self-comfort (prop.) } \\
\hline$M$ & .01 & .03 & .03 & .04 & .05 & .04 \\
\hline$S D$ & .05 & .12 & .10 & .12 & .11 & .11 \\
\hline \multicolumn{7}{|c|}{ Concern } \\
\hline M & 2.28 & 2.48 & 2.37 & 2.30 & 2.81 & 2.56 \\
\hline$S D$ & 1.20 & 1.08 & 1.17 & 0.87 & 1.04 & 0.98 \\
\hline \multicolumn{7}{|c|}{ Global empathy } \\
\hline M & 2.52 & 2.93 & 2.70 & 3.14 & 3.89 & 3.50 \\
\hline$S D$ & 1.19 & 1.20 & 1.20 & 1.27 & 1.30 & 1.30 \\
\hline
\end{tabular}

ous research indicating some differences in physiological functioning between boys and girls (Raine et al., 1997). The analysis of RSA revealed a significant episode effect, $F(1$, $83)=44.50, p<.001$. Across all subjects, mean RSA during resting was $5.82 \ln (\mathrm{ms} 2)$ and decreased to $5.27 \ln (\mathrm{ms} 2)$ during the empathy episode. There were no interactions involving episode, aggression group, and gender. The two risk groups did not differ on resting RSA or RSA during the crying tape ( $M=5.62 \ln (\mathrm{ms} 2)$, resting RSA for aggressive/destructive group, $M=5.89 \operatorname{In}(\mathrm{ms} 2)$, resting RSA for low aggressive/destructive group, $M=5.19 \ln (\mathrm{ms} 2)$, crying tape RSA for aggressive destructive group, $M=5.30$ In(ms2), crying tape RSA for low aggressive/ destructive group).

The analysis of HP indicated main effects for episode $F(1,83)=55.86, p<.001$, and gender, $F(1,83)=6.97, p<.01$. Across all subjects, there was a significant decrease in HP (increase in HR). Mean HP for the resting episode was 550.77 versus $530.23 \mathrm{~ms}$ for the empathy episode. The main effect for gender indicated that, across both resting and empathy episodes, boys displayed greater HP (lower HR) than girls $(M=563.18 \mathrm{~ms}$ for resting and $541.53 \mathrm{~ms}$ for empathy for boys; $M=$ $536.33 \mathrm{~ms}$ for resting and $520.74 \mathrm{~ms}$ for empathy for girls). The two risk groups did not differ on resting HP or HP during the crying tape $(M=$ $553.30 \mathrm{~ms}$, resting HP for aggressive/destructive group, $M=544.77 \mathrm{~ms}$, resting HP for low aggressive/destructive group, $M=531.49 \mathrm{~ms}$, crying tape HP for aggressive/destructive group, $M=529.13 \mathrm{~ms}$, crying tape HP for low aggressive/destructive group).

Relations between physiological measures and empathy measures from Empathy Task 1 (crying tape)

To examine the relation between resting measures of HP and RSA and empathy behaviors, correlations between these two sets of measures were examined while controlling for gender. There was no relation between the two resting measures of cardiac activity and any of the empathy behaviors.

To examine the relation between physiological responding and discrete empathy behaviors, correlations between the RSA and HP change measures and the six measures 
from the crying tape empathy episode were examined while controlling for gender and resting RSA or HP. Changes in HP while hearing the cries of another child were unrelated to empathy behaviors. Higher RSA change scores, indicative of greater decreases in RSA, were correlated with less arousal (freezing, $r=-.28, p<.001)$ and less concern for the victim $(r=-.25, p<.01)$. Thus, children who were better regulated physiologically (i.e., displaying greater RSA suppression during the crying tape) exhibited some indication that they were less empathic.

\section{Discussion}

The goal of this study was to examine the empathy responses of 2-year-old children, some of whom had been identified by parents as displaying problematic levels of aggressive/ destructive behavior. Of primary interest was the behavioral and physiological responses of these children to two empathy-eliciting situations. One situation exposed the children to an emotional display of another child, whom they could hear but not see. The other situation involved observing the pain and distress of the experimenter, whom the children could both see and hear. In addition to examining the children's affective, behavioral, and physiological response to these situations, analyses focused on the relations of these responses to children's aggressive/destructive behavior.

Although empathy behaviors begin to appear during the 2 nd year of life ( $\mathrm{Zahn}-$ Waxier, Radke-Yarrow, et al., 1992), there has been little research investigating empathy's relation to social functioning, specifically aggression, in young children. Empathy and aggression have been linked theoretically and empirically through two different mechanisms. First, both have been associated with poor emotion regulation (Eisenberg et al., 1994, 1996; Young et al., 1999). Just as aggression reflects an inability to control feelings of anger and frustration, responding with personal distress rather than empathy is thought to demonstrate an inability to regulate vicariously induced distress (Eisenberg \& Fabes, 1995; Eisenberg et al., 1994, 1996). Additionally, it has been suggested that low levels of empathy and aggression are both related to a low level of physiological arousal (Young et al., 1999). This study investigated the empathy responses of 2-year-olds and examined affective, behavioral, and physiological measures of arousal and regulation.

Across the two empathy-eliciting events included in this study, there was a consistent pattern of empathy responding for both aggressive/destructive and nonaggressive/destructive toddlers. Concern, hypothesis testing, global empathy, short latencies to respond to the distress, and arousal (only in the first empathy episode) were all significantly related to one another. This pattern is consistent with the findings of Young et al. (1999) and suggests that for a young child, a certain level of arousal, as reflected in muscular tension, is part of an empathic response. Young and colleagues also found that different victims and contexts elicited different levels of various empathic behaviors. Similar results were obtained in the present study, with a greater level of empathic responding occurring in response to the visible distress of an experimenter versus the unseen distress of an unknown child, suggesting that situational variability may be important to take into account when considering how to measure empathy responses in young children. The finding of significant cross-episode correlations for at least some of the measures suggests that there are consistent individual differences in the tendency to display empathic behaviors in response to the distress of another person, regardless of the particular person or context.

In addition to successfully eliciting empathy related behaviors such as arousal, hypothesis testing, and concern, hearing the cries of an unseen child also produced physiological responding. Across all children, there was a significant decrease in both HP (increased HR) and RSA from a resting measure of both variables. This task successfully increased arousal, as indicated by increased HR, and elicited physiological regulation, as indicated by a suppression of RSA. The data from this study demonstrate, consistent with at least two other studies (Young et al., 1999; ZahnWaxier, Radke-Yarrow, et al., 1992), that 
toddlers display affective, behavioral, and physiological indications that they are responsive to, and show concern for, others.

The main question of this study was whether toddlers rated by their mothers as high in aggressive/destructive behavior would display a deficit in empathy skills, including reacting appropriately to the distress of another, becoming appropriately aroused to that distress, displaying concern for the victim, and exploring hypotheses about the source of the victim's distress. Regardless of whether this deficit would be attributed to a lack of arousal or poor regulation, it was expected that 2-year-olds who were high in aggressivedestructive behavior problems would respond with less empathy to the distress displays of others. This prediction was not supported and, in fact, the opposite relation between aggression and empathy behaviors was found. The children in the high-aggression group displayed shorter latencies to respond to the distress of others, engaged in more hypothesis testing, showed greater affective concern, and were rated higher on global empathy than children in the low aggressive/destructive group.

The current findings would seem to indicate that aggressive/destructive children are more empathic than children without externalizing behavior problems. Although this finding may seem counterintuitive, previous studies have suggested that a negative relation between aggression and empathy does not develop until later in development (MacQuiddy et al., 1987; Zahn-Waxler et al., 1995). A recent longitudinal study (Hasting et al., 2000) suggested that this negative relation does not begin to appear until age 6 . Previous studies have found no relation between externalizing behavior and empathy in young children (e.g., Hastings et al., 2000); the current investigation is the only study to date to show a positive relation between empathy behaviors and aggression. Despite these contradictory findings, it is possible that both arousal and regulatory mechanisms play a part in empathy responding.

Different empathy-eliciting situation may tax the child's emotional functioning in different ways. The empathy-eliciting events used in this study, which involved an unknown victim, may have been more challenging to the self-regulation abilities of the 2-year-olds than a situation involving a known victim such as the mother (Young et al., 1999). These situations may prove more challenging in terms of the child's capacity to actually intervene and help, and thus may elicit more negative arousal. If this is the case, we would except toddlers regulating at a developmentally appropriate level, in a situation where they have few skills to intervene, to display more behaviors that focus away from the victim, such as distraction. According to this view, an externalizing child who is not aroused to the victim's distress may appear more empathic because he or she can attend to the distressed other rather than trying to manage his or her own negative arousal. The low-arousal hypothesis also implies that something other than the emotion of empathy is motivating the empathy-related behaviors demonstrated by the aggressive/destructive group-possibly socialization.

A lack of social inhibition, or impulsivity, is often associated with aggressive, externalizing-type behavior (Lewis \& Miller, 1989). The shorter latency to respond and more overt empathic behavior of the aggressive children may, in fact, reflect poorer emotion regulation. In ambiguous situations with unknown or unfamiliar individuals, a well-regulated response with an appropriate level of social inhibition would result in a relative lack of overt behavior. Moreover, externalizing children may be more responsive to the emotions of others because they are more emotionally reactive and less well-regulated in general (Calkins \& Dedmon, 2000). According to Eisenberg and Fabes (1992) higher levels of emotionality are more difficult to regulate. Another explanation for the unexpected findings is that the aggressive children are, in fact, failing to regulate their behavior by behaving impulsively in a situation where their role or responsibility is unclear. Although one interpretation of the muted empathic responses of the nonaggressive-destructive children is that these children may be fearful, there is little support for this hypothesis in the current data. For example, if the nonaggressive group were 
more fearful, higher levels of postural freezing (arousal) would be expected, a pattern that was not found.

The association between a well-regulated emotional response and a more muted empathic response is partially supported by the physiological data in this study. When hearing the distressed cries of an unseen toddler, children displaying greater RSA suppression also displayed less behavioral arousal and less concern. Thus, children who displayed more physiological regulation showed less affective concern for the victim, but they also demonstrated less physical tension (an indicator of a distress or fear response).

Little support was evident for the hypothesis that less physiological arousal would predict less empathy: lower resting HR (higher HP) was not related to any empathy behaviors, nor was there any evidence that aggressive toddlers were less aroused physiologically during the resting episode or the empathy-eliciting event. These findings appear to be inconsistent with studies addressing the relation of physiology and empathy in older children (Eisenberg et al., 1989, 1991). Thus, there is evidence from this and other studies that the pattern of associations between physiology and behaviors is not the same in toddlers as it is in older children. For example, several studies found no relation between early childhood HR and externalizing problems, either predictively (Van Hulle et al., 2000) or concurrently (Calkins \& Dedmon, 2000). These findings, as well as the contradictory behavioral findings, challenge the view that the low empathy, low arousal, high aggression findings observed among older children and adults should necessarily extend downward developmentally. The developmental trajectory may actually be that poor regulation leads to impulsive, aggressive, undercontrolled behavior, then to peer rejection (Coie \& Kuperschmidt, 1983; Dodge, 1983; Panak \& Garber, 1992), and finally to low empathy. Clearly, these findings are in need of replication and further explication, as in the hypothesis that regulatory mechanisms, and not simply arousal, play a role in the development of empathic responding.
Few studies have addressed empathy in young children beyond the description of the progression of typical empathy development. This study adds to the literature addressing individual differences in empathy responding in young children and how these differences are related to factors such as social, emotional, and physiological functioning. Moreover, the findings support the notion that the relation between aggression and empathy behaviors changes through the course of development. The current findings point to some issues that other researchers should focus on when investigating empathy's later relation to aggression. For example, the externalizing children are approaching and even performing rare prosocial behaviors, but at some point these behaviors may stop. These early findings would imply that deficits in empathy responding are not due to a lack of learning, lack of motivation to help others, or temperamentally based lack of attention to others. The RSA data may indicate that under more stressful empathy situations that one would see in real life, the high-risk children may not be able to regulate in order to act. Alternatively, the behavioral indicators of empathy may reflect the influence of another factor other than the emotional experience of empathy, such as impulsivity and socialization. We would argue that the data from this study, although supporting previous research indicating that 2 year-olds are capable of empathic responses, suggests that the current behavioral measures of empathy may not be sensitive enough to distinguish true empathic responding. This view is supported by the physiological data suggesting that the children who are not acting empathic are regulating emotional arousal. Older children and adults, with more sophisticated cognitive and emotion regulation skills, may more readily engage in the kinds of empathy behaviors that seem appropriate. Thus, we might expect to see a reversal of the relation between aggression and empathy at some point in early childhood, when emotion regulation and cognitive monitoring skills have emerged and the effects of peer feedback become part of the process.

This study does have several limitations 
that future investigations should address. One important issue is the validity of the aggressive/nonaggressive classification used in this study. Given that tantrums and acting-out behaviors become more normative during toddlerhood, one might question whether these aggressive/destructive toddlers are, in fact, different in a substantive way from the nonaggressive children. There are several reasons to suggest that these groupings are valid. First, the groupings were based on two administrations of an instrument that has reliably distinguished clinic-referred from non-clinicreferred children (Achenbach, 1992). Second, data from this sample indicates that aggressive/destructive children are physiologically and emotionally less well-regulated and more aggressive with peers (Calkins \& Dedmon; 2000; Calkins, Gill, \& Williford, 1999). The two subscales (aggressive and destructive), which together make up the externalizing scale of the CBCL, were not investigated individually as they relate to empathy behaviors. Although both aggressive and destructive behavior would seem to be indicative of poor regulatory skill, it may be the case that one of these behaviors is more likely to remain consistent beyond toddlerhood and therefore reflects a temperamental level of emotion regulation rather than a transitory behavior associated with this developmental stage. Additionally, if one of these two behaviors (aggressiveness and destructiveness) is driven more by impulsivity than the other, that particular behavior would be responsible for the association between externalizing and empathy. Future investigations should address each of these components individually, as well as other measures of social inhibitions and family factors, such as maternal distress, that were not included in the present study but may have contributed to group differences.

Several other methodological limitations must be mentioned. First, this study does not distinguish empathy form sympathy. Empathy is a vicarious emotion similar to that of the victim, in this case sadness or pain from injury, whereas sympathy is concern for the victim. There is a meaningful difference between these two constructs, but the difference is difficult to measure in observational studies of negative vicarious emotion. Due to the young age of the participants and the fact that the victims were either unseen or an unfamiliar adult, empathy behavior may have been significantly reduced and thus not accurately reflected the empathic abilities of well-regulated low aggressive/destructive children. Future investigations should attempt to replicate these findings in more ecologically sensitive contexts, such as familiar peer groups. A second limitation of the study is that only one source of information, maternal report, was used to classify children in externalizing groups. Although grouping was based on repeated maternal report and these children differed on several other measures beyond empathy (Calkins \& Dedmon, 2000; Calkins, Gill, \& Williford, 1999), the reports of a day care teacher or other child care provider would have provided a confirmation of child externalizing behavior problems. Moreover, there is good evidence that behavior problems of this sort are only modestly stable prior to preschool. A final limitation in the methodology is the fact that there were two experimenters who feigned distress. There may have been small variations in examiner performance that contributed to variations in participant response.

Future studies should address the consistency of empathic behavior for aggressive and nonaggressive children across contexts. A situation in which a child must regulate emotional responsivity to the distress of a loved one or to a victim whom he or she has injured may be more taxing to the emotional system of a young child and lead to a lack of empathy resulting from emotional overarousal. Longitudinal studies will illuminate further the developing relation between empathy and aggression. Nevertheless, the data from the present study suggest that the link between aggression and empathy may be more complicated than previously thought and may be influenced by developmental changes in the magnitude of responsivity to the emotions of others and social factor influencing this responsivity. 


\section{References}

Achenbach, T. M. (1992). Manual for the child behavior checklist/2-3 and 1992 profile. Burlington, VT: University of Vermont, Department of Psychiatry.

Achenbach, T. M., Edelbrock, C., \& Howell, C. T. (1987). Empirically based assessment of the behavioral/emotional problems of 2- and 3-year-old children. Journal of Abnormal Child Psychology, 15, 629-650.

American Psychological Association. (1994). Diagnostic and statistical manual of mental disorders (4th ed.). Washington, DC: Author.

Bates, J. E., Bayles, K., Bennet, D. S., Ridge, B., \& Brown, M. (1991). Origins of externalizing behavior problems at eight years of age. In D. Pepler \& K. Rubin (Eds.), The development and treatment of childhood aggression (pp. 93-120). Hillsdale, NJ: Erlbaum.

Batson, C. D. (1991). The altruism question: Toward a social psychological answer. Hillsdale, NJ: Erlbaum.

Belsky, J., Woodworth, S., \& Crnic, K. (1996). Troubled family interaction during toddlerhood. Development and Psychopathology, 8, 477-495.

Cacioppo, J. T., \& Sandman, C. A. (1978). Physiologica differentiation of sensory and cognitive tasks as a function of warning processing demands and reported unpleasantness. Biological Psychology, 6, 181-192.

Calkins, S. D. (1997). Cardiac vagal tone indices of behavioral reactivity and regulation in young children. Developmental Psychobiology, 31, 125-135.

Calkins, S. D., \& Dedmon, S. A. (2000). Physiological and behavioral regulation in two-year-old children with aggressive/destructive behavior problems. Journal of Abnormal Child Psychology, 28, 103-118.

Calkins, S. D., Gill, K., Smith, C., \& Johnson, M. C. (1999). Emotional reactivity and emotion regulation strategies as predictors of social behavior with peers during toddlerhood. Social Development, 8, 310-341.

Calkins, S. D., Gill, K., \& Williford, A. (1999). Social behavior and peers' responses to aggression. Early Education and Development, 10, 267-288.

Campbell, S. B. (1990). Behavior problems in preschool children: Clinical and developmental issues. New York: Guilford Press.

Campbell, S. B. (1997). Behavior problems in preschoo children: Developmental and family issues. Advances in Clinical Child Psychology, 19, 1-26.

Cicchetti, D., Ganiban, J., \& Barnett, D. (1991). Contributions from the study of high-risk populations to understanding the development of emotion regulation. In J. Garber \& K. A. Dodge (Eds.). The development of emotion regulation and dysregulation (pp. 15-48) Cambridge: Cambridge University Press.

Coie, J. D., \& Kuperschmidt, J. B. (1983). A behaviora analysis of emerging social status in boys' groups. Child Development, 54, 1400-1416.

Davis, M. H. (1994). Empathy: A social psychological approach. Madison, WI: Brown \& Benchmark.

Dodge, K. A. (1983). Behavioral antecedents of peer social status. Child Development, 53, 1386-1399.

Edwards, C. P. (1995). Parenting toddlers. In M. H. Bornstein (Ed.), Handbook of parenting: Vol. 1. Children and parenting (pp. 41-63). Hillsdale, NJ: Erlbaum.

Eisenberg, N., \& Fabes, R. A. (1990). Empathy: Conceptualization, measurement, and relation to prosocial behavior. Motivation and Emotion, 14, 131-149.
Eisenberg, N., \& Fabes, R. (1992). Emotion, regulation, and the development of social competence. In M. S. Clark (Ed.), Review of personality and social psychology: Vol. 14. Emotion and social behavior (pp. 119150). Thousand Oaks, CA: Sage.

Eisenberg, N., \& Fabes, R. A. (1995). The relation of young children's vicarious emotional responding to social competence, regulation, and emotionality. Cognition and Emotion, 9, 203-229.

Eisenberg, N., \& Fabes, R. (1998). Prosocial development. In W. Damon (Series Ed.) \& N. Eisenberg (Volume Ed.), Handbook of child psychology: Vol 3. Social, emotional and personality development (5th ed., pp. 177-235). New York: Wiley.

Eisenberg, N., Fabes, R. A., Miller, P. A., Fultz, J., Shell, R., Mathy, R., \& Reno, R. R. (1989). Relation of sympathy and personal distress to prosocial behavior: A multimethod study. Journal of Personality and Social Psychology, 57, 55-66.

Eisenberg, N., Fabes, R. A., Murphy, B., Karbon, M., Smith, M., \& Maszk, P. (1996). The relations of children's dispositional empathy-related responding to their emotionality, regulation, and social functioning. Developmental Psychology, 32, 195-209.

Eisenberg, N., Fabes, R. A., Murphy, B., Karbon, M. Maszk, P., Smith, M., O'Boyle, C., \& Suh, K. (1994). The relations of emotionality and regulation to dispositional and situational empathy-related responding. Journal of Personality and Social Psychology, 66, 776-797.

Eisenberg, N., Fabes, R. A., Schaller, M., Carlo, G., \& Miller, P. A. (1991). The relations of parental characteristics and practices to children's vicarious emotional responding. Child Development, 62, 13931408.

Eisenberg, N., \& Miller, P. (1987). The relation of empathy to prosocial and related behaviors. Psychological Bulletin, 101, 91-119.

Fabes, R. A., Eisenberg, N., Karbon, M., Troyer, D., \& Switzer, G. (1994). The relations of children's emotion regulation to their vicarious emotional responses and comforting behaviors. Child Development, 65, 1678-1693.

Fox, N. A. (1989). Psychophysiological correlates of emotional reactivity during the first year of life. Developmental Psychology, 25, 364-372.

Fox, N. A., Schmidt, L. A., Calkins, S. D., Rubin, K. H., \& Coplan, R. J. (1996). The role of frontal activation in the regulation and dysregulation of social behavior during the preschool years. Development and Psychopathology, 8, 89-102.

Guthrie, I. K., Eisenberg, N., Fabes, R. A., Murphy, B. C., Holmgren, R., Mazsk, P., \& Suh, K. (1997). The relations of regulation and emotionality to children's situational empathy-related responding. Motivation and Emotion, 21, 87-108.

Hastings, P. D., Zahn-Waxler, C. R., Robinson, J. Usher, B., \& Bridges, D. (2000). The development of concern for others in children with behavior problems. Developmental Psychology, 36, 531-546.

Hoffman, M. L. (1982). Development of prosocial motivation: Empathy and guilt. In N. Eisenberg (Ed.), The development of prosocial behavior (pp. 218-231). San Diego, CA: Academic Press.

Howes, C., \& Farver, J. (1987). Toddlers' response to the 
distress of their peers. Journal of Applied Developmental Psychology, 8, 441-452.

Hubbard, J., \& Coie, J. D. (1994). Emotional determinants of social competence in children's peer relationships. Merrill-Palmer Quarterly, 40, 1-20.

Keenan, K., \& Shaw, D. (1997). Developmental and social influences of young girls' early problem behavior. Psychological Bulletin, 121, 95-113.

Kopp, C. (1982). Antecedents of self-regulation: A developmental perspective. Developmental Psychology, 18, 199-214.

Lewis, M., \& Miller, S. (1989). Handbook of developmental psychopathology. New York: Plenum Press.

MacQuiddy, S. L., Maise, S. J., Hamilton, S. B. (1987). Empathy and affective perspective-taking skills in parent-identified conduct-disordered boys. Journal of Clinical Child Psychology, 16, 260-268.

Martin, G. B., \& Clark, R. D. (1982). Distress crying in neonates: Species and peer specificity. Developmental Psychology, 18, 3-9.

Miller, P., \& Eisenberg, N. (1988). The relation of empathy to aggressive and externalizing/antisocial behavior. Psychological Bulletin, 103, 324-344.

Panak, W. F., \& Garber, J. (1992). Role of aggression, rejection, and attributions in the predictions of depression in children. Development and Psychopathology, 4, 145-165.

Porges, S. W. (1985). U.S. Patent No. 4520944. Washington, DC: U.S. Patent and Trademark Office.

Porges, S. W. (1991). Vagal tone: An autonomic mediator of affect. In J. Garber \& K. A. Dodge (Eds.) The development of emotional regulation and dysregulation (pp. 111-128). Cambridge: Cambridge University Press.

Porges, S. W. (1996). Physiological regulation in highrisk infants: A model for assessment and potential intervention. Development and Psychopathology, 8, 43-58.
Porges, S. W., Doussard-Roosevelt, J. A., \& Maiti, A. K. (1994). Vagal tone and the physiological regulation of emotion. Monograph of the Society for Research in Child Development, 59, 167-186.

Raine, A., Venables, P., \& Mednick, S. (1997). Low resting heart rate at age three years predisposes to aggression at age 11 years: Evidence from the Mauritius Child Health Project. Journal of the Academy of Child and Adolescent Psychiatry, 36, 1457-1464.

Ruff, H., \& Rothbart, M. K. (1996). Attention in early development. New York: Oxford University Press.

Sagi, A., \& Hoffman, M. L. (1976). Empathic distress in newborns. Developmental Psychology, 12, 175-176.

Sroufe, A. (1996). Emotional development. Cambridge: Cambridge University Press.

Thomas, R. A., \& Calkins, S. D. (1996). The doubleedged sword: Emotional regulation for children at risk. Development and Psychopathology, 8, 163-182.

Van Hulle, C. A., Corley, R., Zahn-Waxler, C., Kagan, J., \& Hewit, J. K. (2000). Early childhood heart rate does not predict externalizing behavior problems at age 7 years. Journal of the American Academy of Child and Adolescent Psychiatry, 39, 1287-1344.

Young, S. K., Fox, N. A., \& Zahn-Waxler, C. (1999). The relations between temperament and empathy in 2-year-olds. Developmental Psychology, 35, 11891197.

Zahn-Waxler, C., Cole, P. M., Welsh, J. D., \& Fox, N. A. (1995). Psychophysiological correlates of empathy and prosocial behaviors in preschool children with behavior problems. Development and Psychopathology, 7, 27-48.

Zahn-Waxler, C., Radke-Yarrow, M., Wagner, E., \& Chapman, M. (1992). Development of Concern for Others. Developmental Psychology, 28, 126-136.

Zahn-Waxler, C., Robinson, J. L., \& Emde, R. N. (1992). The development of empathy in twins. Developmental Psychology, 28, 1038-1047. 